\title{
Plane mixing layer simulation using FEniCS libraries
}

\author{
Diego R. González* \\ National University of Asuncion, Chemical Engineering Department \\ San Lorenzo, PY \\ drweiberlen@gmail.com. \\ Hyun H. Shin Christian E. Schaerer \\ National University of Asuncion, LCCA \\ San Lorenzo, PY \\ hhs82528@gmail.com_chris.schaerer@gmail.com
}

\begin{abstract}
$\underline{\text { ABSTRACT }}$ for example, combustion, and transport and mixing process of chemical substances [5]. dimensional flow are reduced to:

$$
\begin{aligned}
\frac{\partial u}{\partial x}+\frac{\partial v}{\partial y} & =0 \\
\frac{\partial u}{\partial t}+u \frac{\partial u}{\partial x}+v \frac{\partial u}{\partial y} & =-\frac{1}{\rho} \frac{\partial p}{\partial x}+\nu\left(\frac{\partial^{2} u}{\partial x^{2}}+\frac{\partial^{2} u}{\partial y^{2}}\right) \\
\frac{\partial v}{\partial t}+u \frac{\partial v}{\partial x}+v \frac{\partial v}{\partial y} & =-\frac{1}{\rho} \frac{\partial p}{\partial y}+\nu\left(\frac{\partial^{2} v}{\partial x^{2}}+\frac{\partial^{2} v}{\partial y^{2}}\right)
\end{aligned}
$$
\end{abstract}

The plane mixing layer is a common phenomena encountered in many engineering applications [3],

The mixing layer is produced by the interaction of two parallel fluid streams, away from the wall, with different velocities $U_{2}>U_{1} \geq 0$ [6]. Due to the velocity differences in the interface, the shear stress is generated producing the Kelvin-Helmholtz instability [3], which is characterized for the enrolled vortex structures enhancing the momentum, heat and mass transfer. The plane mixing layer is considered a bi-dimensional flow, but the turbulences present in the flow have tri-dimensional characteristics [7].

The aim of this work is to evaluate the bi-dimensional numerical simulation of plane mixing layer on a refined mesh. A reduced computational domain is used in order to obtain an initial result. The averaged velocity profile is compared to the results from the experiments [1]. The FEniCS Project [4] is used for the numerical simulation, specially the CBC.PDESys package which is an application of FEniCS written in Python for the resolution of partial differential equations for fluid dynamics using finite element method. The flow is considered incompressible, and time dependent perturbations [2] are applied in order to model the instability inherent to the real phenomena. The dominant direction of flow is $x$, the variations of flow are predominately in the $y$ direction, and it is homogeneous and symmetric in $z$ direction. The flow is modelled by the continuity and Navier-Stokes equations. These equations in two

where, $u, v$ are velocities component in the flow direction and transversal direction, respectively, $p$ denotes pressure, and $\nu$ the kinematic viscosity. The boundary conditions imposed are symmetry on top and bottom for the velocity, and constant pressure in the outlet. The velocities in the inlet are $U_{2}=1.5$ and $U_{1}=0.5$, the dimensionless velocity is $\left(\frac{U-U_{1}}{U_{2}-U_{1}}\right)$, where the velocity $U$ represent the time averaged of $u$ at the distance $x=50$ (center of domain), and the $y$ coordinate is normalized by the

\footnotetext{
${ }^{*}$ scientific initiation student Faculty of Chemical Sciences/National University of Asuncion
} 
vorticity thickness $\delta_{v}=\left(\left.\frac{\partial U}{\partial y}\right|_{\max }\right)^{-1}$. The computational domain is $0 \leq x \leq 100$ and $-15 \leq y \leq 15$ and the mesh consists in $201 \times 61$ elements. The simulation time is long enough to reach the steady-state.

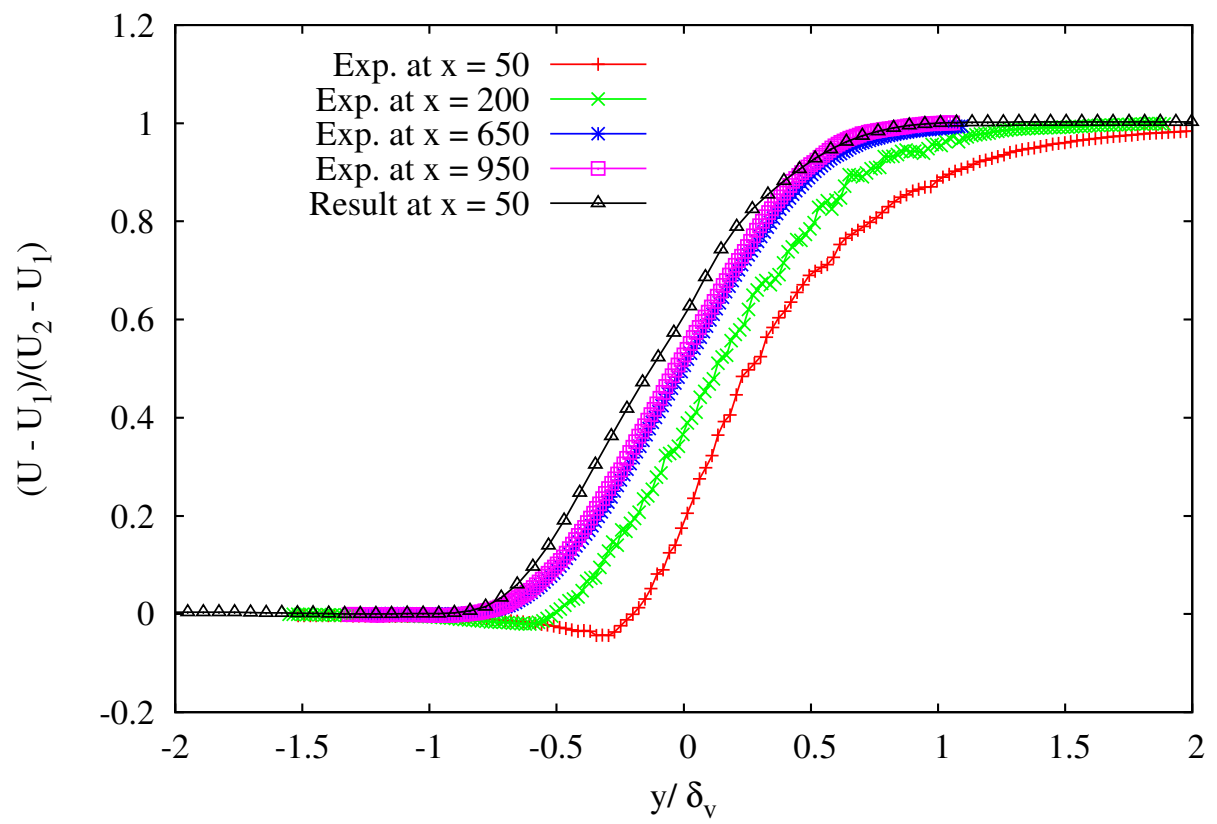

The Figure shows the comparison of the average velocity $U$ obtained from numerical simulation with experimental data from [1]. The axes $x$ and $y$ represent the dimensionless transversal direction and the dimensionless velocity, respectively. The average velocity is computed after the steady-state is reached. The numerical result and the experimental data are in good agreement.

The initial result obtained from the numerical simulation of plane mixing layer is promising. However, further studies should be performed with larger computational domains, and also, more turbulence statistics should be computed and analyzed.

Palabras-clave: Plane mixing layer, Kelvin-Helmholtz instability, FEniCS Project, Finite element method

\section{BIBLIOGRAPHY}

[1] J. Delville, S. Bellin, J. H. Garem, \& J. P. Bonnet, Analysis of structures in a turbulent, plane mixing layer by use of a pseudo flow visualization method based on hot-wire anemometry, In Advances in Turbulence 2 (1989) (251-256), Springer Berlin Heidelberg.

[2] O. Inoue, Note on multiple-frequency forcing on mixing layers, Fluid Dynamics Research, (1995) 16(2):161-172.

[3] P. Kundu \& I. Cohen, Fluid Mechanics, Elsevier Science, 2010.

[4] A. Logg, K. Mardal, \& G. Wells, Automated Solution of Differential Equations by the Finite Element Method: The FEniCS Book, Lecture Notes in Computational Science and Engineering, Springer, 2012.

[5] M. J. Maghrebi \& A. Zarghami, DNS OF FORCED MIXING LAYER, International Journal of Numerical Analysis \& Modeling, (2010) 7(1):173-193.

[6] S. Pope, Turbulent Flows, Cambridge University Press, 2000.

[7] H. Tennekes \& J. Lumley, A First Course in Turbulence, MIT Press, 1972. 